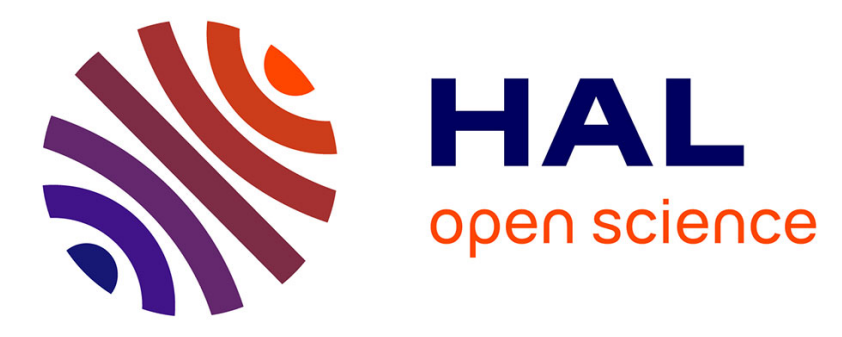

\title{
Cortical motor output decreases after neuromuscular fatigue induced by electrical stimulation of the plantar flexor muscles
}

Francois Alexandre, Gerard Derosiere, Maria Papaiordanidou, Maxime Billot, Alain Varray

\section{To cite this version:}

Francois Alexandre, Gerard Derosiere, Maria Papaiordanidou, Maxime Billot, Alain Varray. Cortical motor output decreases after neuromuscular fatigue induced by electrical stimulation of the plantar flexor muscles. Acta Physiologica, 2015, 214 (1), pp.124-134. 10.1111/apha.12478 . hal-01622341

\section{HAL Id: hal-01622341 \\ https://hal.umontpellier.fr/hal-01622341}

Submitted on 10 Nov 2017

HAL is a multi-disciplinary open access archive for the deposit and dissemination of scientific research documents, whether they are published or not. The documents may come from teaching and research institutions in France or abroad, or from public or private research centers.
L'archive ouverte pluridisciplinaire HAL, est destinée au dépôt et à la diffusion de documents scientifiques de niveau recherche, publiés ou non, émanant des établissements d'enseignement et de recherche français ou étrangers, des laboratoires publics ou privés. 


\section{Cortical motor output decreases after neuromuscular fatigue induced by electrical stimulation of the plantar flexor muscles}

François Alexandre ${ }^{\mathrm{a}, \mathrm{b}}$, Gerard Derosiere ${ }^{\mathrm{a}, \mathrm{c}, 2}$, Maria Papaiordanidou ${ }^{\mathrm{a}, \mathrm{d}}$, Maxime Billot ${ }^{\mathrm{a}, 1}$, Alain Varray $^{\text {a }}$

\section{Affiliations:}

a, Movement To Health, Euromov, Montpellier University, Montpellier, France

b, Clinique du Souffle "la Vallonie", Fontalvie, Lodève, France

c, Biomedical Engineering Research Group, National University of Ireland, Maynooth, Co Kildare, Ireland

d, Institut des Sciences du Mouvement, Aix-Marseille University, Marseille, France

\section{Corresponding author}

François Alexandre, Movement to Health, Faculty of Sports Sciences, Montpellier University, Euromov, 700 Avenue du Pic Saint Loup, 34090, Montpellier, France.

Tel.: + 33. (0)434.432.632; Fax: + 33. (0)434.432.644

E-mail: francois.alexandre@fontalvie.fr

\section{Present adress:}

1, Faculté de médecine, Département de kinésiologie, Université Laval, Québec, Canada

2, Cognition and Actions Laboratory, Institute of Neuroscience, Université catholique de Louvain, Brussels, Belgium

Short title: Cortical motor output after electrically induced fatigue 


\section{Abstract}

Aim: Neuromuscular electrical stimulation (NMES) causes early onset of neuromuscular fatigue. Peripheral electrophysiological explorations suggest that supra-spinal alterations are involved through sensitive afferent pathways. As sensory input is projected over the primary somatosensory cortex (S1), S1 area involvement in inhibiting the central motor drive can be hypothesized. This study assessed cortical activity under a fatiguing NMES protocol at low frequency.

Methods: Twenty healthy males performed five NMES sequences of 17 trains over the plantar flexors $(30 \mathrm{~Hz}, 4 \mathrm{~s}$ on/6s off). Before and after each sequence, neuromuscular tests composed of maximal voluntary contractions (MVCs) were carried out. Cortical activity was assessed during MVCs with functional near-infrared spectroscopy over S1 and primary motor (M1) areas, through oxy- $[\mathrm{HbO}]$ and deoxy-hemoglobin $[\mathrm{HbR}]$ variation. Electrophysiological data (H-reflex during MVC, EMG activity and level of voluntary activation) were also recorded.

Results: MVC torque significantly decreased after the first 17 NMES trains $(p<0.001)$. The electrophysiological data were consistent with supra-spinal alterations. In addition, [HbO] declined significantly during the protocol over the S1 and M1 areas from the first 17 NMES trains $(p<0.01$ and $p<0.001$, respectively), while $[\mathrm{HbR}]$ increased $(p<0.05$ and $p<0.01$, respectively), indicating early decline in cortical activity over both primary cortical areas.

Conclusions: The declining cortical activity over the M1 area is highly consistent with the electrophysiological findings and supports motor cortex involvement in the loss of force after a fatiguing NMES protocol. In addition, the declining cortical activity over the S1 area indicates that the decreased motor output from M1 is not due to increased S1 inhibitory activity. 
Key words: Neuromuscular electrical stimulation, supra-spinal fatigue, central motor drive, fNIRS 


\section{Introduction}

2 Neuromuscular electrical stimulation (NMES) is a peripheral type of solicitation that consists

3 of setting off a muscular contraction electrically by activating intramuscular nerve branches

4 (Hultman et al., 1983). Despite widespread clinical use, NMES presents a strong limitation: it

5 provokes rapid and excessive neuromuscular fatigue (Doucet et al., 2012, Downey et al.,

6 2011). In rehabilitation, low stimulation frequencies are often preferred as they delay the

7 development of neuromuscular fatigue compared with high stimulation frequencies (Gorgey

8 et al., 2009). Therefore, previous investigations of NMES-induced neuromuscular fatigue

9 were conducted at low frequencies, notably over the plantar flexors (Papaiordanidou et al., 2010b) and the thumb abductor muscle (Papaiordanidou et al., 2010a). Opposite effects were

11 reported, depending on the nature of the stimulated muscle: whereas NMES applied over the high fatigue-resistant plantar flexor muscles mainly induced supra-spinal alterations (Papaiordanidou et al., 2010b), the authors failed to report the same effects over the low fatigue-resistant thumb abductor muscle (Papaiordanidou et al., 2010a). In both studies, given the preservation of spinal excitability, the presence or absence of supra-spinal alterations were deduced at the peripheral level through (i) the level of voluntary activation (LOA) and (ii) the root mean square (RMS) of the electromyographic (EMG) activity normalized by the maximal M-wave $\left(\mathrm{RMS}_{\mathrm{EMG}} / \mathrm{M}\right)$, two markers that may lead to misinterpretation. Indeed, LOA sometimes overestimates the neural drive (Kooistra et al., 2007), while $\mathrm{RMS}_{\mathrm{EMG}}$ may underestimate it because of the amplitude cancellation phenomenon (Keenan et al., 2005).

21 Therefore, if based only on the use of peripheral markers, the existence of supra-spinal 22 alterations in electrically induced fatigue remains mainly speculative.

23 The complementary use of neuroimaging techniques like functional near infrared spectroscopy (fNIRS) or functional magnetic resonance imaging (fMRI) could resolve this issue. Previous studies have reported a decline in cortical activity during volitional fatigue 
using fNIRS (Shibuya \& Tachi, 2006) and fMRI (Liu et al., 2002), suggesting the relevance of such techniques to explore the neurophysiological mechanisms associated with the fatigue phenomenon.

One might therefore wonder which brain areas would be of interest in a neuroimaging-based investigation of electrically induced neuromuscular fatigue. The primary motor area (M1) is incontestably of relevance in this context as it represents the main motor output from the cortex to the spinal cord. Second, the primary somatosensory cortex (S1) is a candidate as it houses projections from the group III and IV afferents (Almeida et al. 2004; Chen et al. 2009; Kalliomaki et al. 1998; Schouenborg et al. 1986), which are strongly suspected of involvement in the neuromuscular fatigue under NMES (Boerio et al. 2005; Papaiordanidou et al. 2010b). The potential involvement of the S1 area in central motor drive inhibition is supported by several studies reporting increased activity in this area during electrical nerve stimulation (Forss et al., 1994, Hlushchuk \& Hari, 2006). Also, the increase in S1 activity was found to occur in parallel to a decrease in primary motor cortex (M1) activity (Chen et al., 1999, Hlushchuk \& Hari, 2006) and excitability (Chen et al., 1999, Tokimura et al., 2000). Accordingly, an inhibitory influence exerted by the S1 area toward the M1 area has been hypothesized (Sutherland, 2006).

Besides the potential involvement of the S1 area in decreasing motor output, many studies have reported prefrontal cortex (PFC) deactivation during volitional fatigue just before task failure (Shibuya et al., 2004, Rupp \& Perrey, 2008). As the PFC area is the main facilitation structure of the brain (Tanaka \& Watanabe, 2012), it seems likely that it would be implicated in non-volitional fatigue, although this has not yet been investigated.

To contribute to a better understanding of the neural substrates of NMES-induced neuromuscular fatigue, the present study aimed to (i) attest the supra-spinal alterations by cortical measurements and (ii) test for S1 area involvement in the decrease in motor output 
51 after a fatiguing NMES task at low frequencies over the plantar flexor muscles. We hypothesized an increase in S1 area activity, a decrease in M1 activity and central motor drive failure after electrically induced neuromuscular fatigue.

\section{Materials and methods}

\subsection{Participants}

Twenty healthy males (mean age $23.9 \pm 2.4$ years) participated in the study. They were all physically active, with no history of lower limb surgery or recent injury and no neurological, endocrine, respiratory, or cardiovascular disease or medication use that could affect brain function or perfusion. After being informed about the objectives and potential risks of participation in the study, they all gave written consent. They were asked to avoid any violent effort during the two days before the experimental session. All procedures were approved by the local Ethics Committee and complied with the principles of the Declaration of Helsinki for human experimentation.

\subsection{Pre-experiment}

Nine participants were tested in a control session 7 to 10 days prior to the experiment. During this control session, the settings and the protocol were strictly the same as for the experimental session (as detailed below), except that the NMES sequences (fatigue protocol) were replaced by resting periods that lasted 3 minutes.

\subsection{Experimental design}

The experimental session began with a standard warm-up consisting of 12 submaximal plantar flexions for $2 \mathrm{~s}$ every $5 \mathrm{~s}$, and then six maximal plantar flexions every $30 \mathrm{~s}$. The 
participants were also familiarized with superimposed evoked potentials at this time. Familiarization to maximal plantar flexions was assumed to be sufficient when task criteria were reached (quick rise in force, maintenance of a plateau, absence of compensatory movements). The protocol began with a 3-min resting period to stabilize the participant's physiological variables. The fatigue protocol consisted of five sequences of 17 stimulation trains of the plantar flexor muscles, each lasting $3 \mathrm{~min}$. Neuromuscular tests were performed before and after each stimulation sequence in order to obtain a follow-up before (pre), during (post17, post34, post51, post68), and after (post85) the protocol. The design is presented in Fig. 1A.

\subsection{Fatigue protocol}

The plantar flexor muscles of the dominant leg, formed by the gastrocnemii medialis and lateralis and the soleus muscles, were electrically stimulated by a constant-current stimulator (Cefar Physio 4, Cefar Medical, Lund, Sweden) delivering rectangular symmetric, biphasic pulses at maximal tolerated intensity. The two rectangular $50-\mathrm{cm}^{2}$ surface electrodes (Medicompex, Ecublens, Switzerland) were respectively placed $5 \mathrm{~cm}$ under the popliteal fossa and at the level of the insertion point of the two gastrocnemii muscles on the Achilles tendon. Train characteristics were the following: frequency $30 \mathrm{~Hz}$, pulse duration $450 \mu \mathrm{s}$, duty cycle $40 \%$ ( $4 \mathrm{~s}$ on, $6 \mathrm{~s}$ off), and intensity: maximal tolerated, readjusted at the beginning of each sequence to reach the maximal tolerated threshold continuously (Maffiuletti, 2010). The mean intensity was $33.83 \pm 7.7 \mathrm{~mA}$. The mean percentage of maximal voluntary torque evoked by the stimulation trains was $44.06 \pm 13.82 \%$. 


\subsubsection{Mechanical recording}

104 Plantar flexion torque during voluntary and electrically induced contractions of the dominant

105 leg was recorded by a Biodex System 3 isokinetic dynamometer coupled with the Biodex

106 Advantage Software Package (Medical System Inc., Shirley, NY, USA). The dominant leg was determined using the balance recovery test (Hoffman et al., 1998). The participant was comfortably seated on the Biodex chair in a semi-lying position, with a $30^{\circ}$ back inclination.

109 Chair adjustments were made to line up the foot, the patella and the coxofemoral articulation 110 in the same axis. The ankle and knee angles were respectively set to $90^{\circ}$ and $110^{\circ}\left(180^{\circ}\right.$ full

111 extension) and the dynamometer axis was aligned with the anatomical ankle plantar- and

112 dorsi-flexion axis. The pelvis and the proximal extremity of the patella were securely attached 113 to the chair to minimize the movements of adjacent muscles.

\subsubsection{EMG recording}

116 The surface EMG signals of the soleus and antagonist tibialis anterior muscles were recorded

117 using bipolar, silver chloride, square surface electrodes with a 9-mm diameter (Contrôle

118 Graphique Médical, Brie-Compte-Robert, France). In order to minimize impedance $(<5 \mathrm{k} \Omega)$,

119 the skin was shaved, abraded, and cleaned with alcohol. After verification of an appropriate

120 M-wave acquisition (single response and highest amplitude), electrodes were placed over the 121 muscle belly with an interelectrode distance of $20 \mathrm{~mm}$. The reference electrode was placed on

122 the opposite wrist. The EMG signal was amplified (gain $\times 1000)$, bandpass-filtered (10-500

$123 \mathrm{~Hz}$ ) and recorded at a sampling frequency of $4096 \mathrm{~Hz}$ (Biopac MP100, Biopac Systems, Santa 124 Barbara, CA, USA). 
127 Muscle excitability was evaluated by the superimposed M-wave (Msup), spinal excitability by 128 the superimposed H-reflex (Hsup), and spinal and supra-spinal adaptations by the volitional wave (V-wave). These three evoked potentials were assessed during plantar flexor maximal voluntary contraction (MVCs) by stimulation of the tibial nerve with a constant-current, high

131 voltage stimulator (DS7AH, Digitimer, Hertforshire, UK). Rectangular monophasic pulses of $132200 \mu \mathrm{s}$ were used (Papaiordanidou et al., 2010b). The cathode (a silver chloride square 133 surface electrode) was fixed on the popliteal fossa. A piece of foam, sustained by a strap, was 134 applied over the cathode to optimize contact with the tibial nerve, situated in depth. The anode 135 (a rectangular $50 \mathrm{~cm}^{2}$ surface electrode) was placed beneath the patella. After localization of 136 the optimal site for tibial nerve stimulation, where no response could be observed on the 137 antagonist tibialis anterior, the M-wave and H-reflex recruitment curves were performed at rest. One pulse was delivered on the tibial nerve every $10 \mathrm{~s}$ with the intensity increasing from

$13910 \mathrm{~mA}$ until no further increase in twitch mechanical response and M-wave amplitude 140 occurred. To ensure that M-wave was maximal during MVCs, the maximal intensity at which 141 maximal M-wave amplitude was reached was increased by $20 \%$ (supramaximal intensity,

$\left.142 \mathrm{I}_{\mathrm{Mmax}}\right)$ to account for activity-dependent changes in motor axonal excitability (Racinais et al., 143 2013). This intensity was used during MVCs to obtain Msup and V-wave. Subsequently to $144 \mathrm{I}_{\mathrm{Mmax}}$ determination, the intensity where the maximum H-reflex was observed was carefully 145 sought $\left(\mathrm{I}_{\mathrm{H} \max }\right)$ using 2-mA increments. $\mathrm{I}_{\mathrm{Hmax}}$ was used during MVCs to obtain Hsup.

147 2.5.4. Cortical activity recording

148 Cortical activity was assessed with a continuous wave multichannel functional near-infrared 149 spectroscopy (fNIRS) system (Oxymon Mark III, Artinis, the Netherlands) as described 150 elsewhere (Derosiere et al., 2014). Briefly, it is based on neurovascular coupling: when neural 
activity increases, the increase in regional cerebral blood flow is ten times higher than the

152 increase in regional oxygen consumption, leading to local hyperoxygenation (Fox et al.,

153 1988), and a subsequent increase in fNIRS-measured oxy-hemoglobin ( $\mathrm{HbO})$ with a decrease

154 in fNIRS-measured deoxy-hemoglobin (HbR) (Colier et al., 1999). The fNIRS optodes were

155 positioned over the scalp in accordance with the extended modified international EEG 10-10

156 systems (Fig. 2). One receptor and four emitters were placed around $\mathrm{C}_{3}$ and $\mathrm{CP}_{3}$ (left

157 hemisphere) for right leg stimulation or $\mathrm{C}_{4}$ and $\mathrm{CP}_{4}$ (right hemisphere) for left leg stimulation

158 at a $3.5-\mathrm{cm}$ interoptode distance. This probe arrangement allowed us to target the contralateral

159 (to the stimulated dominant leg) primary motor cortex (M1) and the contralateral

160 somatosensory cortex (S1), through the rostral and caudal parts, respectively. Four recording

161 paths were obtained: two over M1 and two over S1. To target the contralateral PFC area, one

162 receptor and one emitter were also placed around Fp1 (left hemisphere) for stimulation of the

163 right leg or Fp2 (right hemisphere) for stimulation of the left leg. fNIRS data were corrected

164 by implementing a specific differential pathlength factor $\left(4.99+0.067 \times\right.$ age $\left.^{0.814}\right)($ Duncan et

165 al., 1996), which yielded more accurate measurement of the concentration changes,

166 converting the concentration changes in $[\mathrm{HbO}]$ and $[\mathrm{HbR}]$ to $\mu \mathrm{M}$ units (Delpy et al., 1988).

167 The sampling frequency was set at $10 \mathrm{~Hz}$. The NIRS signals were low-pass filtered (finite

168 impulse response) with a cut-off frequency of $0.7 \mathrm{~Hz}$ to remove heart rate signal (Huppert et

169 al., 2009). Specific events were set in the Oxysoft software (V6.0, Artinis, the Netherlands) to

170 distinguish the beginning and the end of each MVC.

171

172 2.6. Neuromuscular tests

173 The neuromuscular tests were conducted during two MVCs with electrically evoked

174 potentials, voluntary activation assessment, and measurement of cerebral oxygenation. Each

175 subject was verbally encouraged during each MVC, which lasted for 4 s. During the plateau 
176 of the first MVC, a single stimulation at $\mathrm{I}_{\mathrm{Mmax}}$ was first delivered. Immediately after, a

177 superimposed doublet $(100 \mathrm{~Hz})$ at $\mathrm{I}_{\mathrm{Mmax}}$ followed, as well as a control doublet $2 \mathrm{~s}$ after the 178 relaxation, according to the twitch interpolation technique (Allen et al., 1995). During the

179 plateau of the second MVC, a single stimulation at $\mathrm{I}_{\mathrm{Hmax}}$ was delivered. The MVCs were

180 separated by a 1-min resting period, during which three resting stimulations at $\mathrm{I}_{\mathrm{Mmax}}$ were

181 delivered to calculate the mechanical response $(\mathrm{Pt})$. The design of the neuromuscular tests is 182 presented in Fig. 1B.

\subsection{Data analysis}

185 The peak of the mechanical response $(\mathrm{Pt})$ during the three resting evoked potentials of the

186 neuromuscular tests was calculated and averaged. The torque evoked by the stimulation trains

187 was recorded and averaged for the first three trains of the first NMES sequence (trains 1-3)

188 and for the last three trains of each NMES sequence (trains 15-17, 32-34, 49-51, 66-68 and

189 83-85) in order to obtain muscle response to the stimulation trains. For each block of 190 neuromuscular tests, the highest torque plateau of the two MVCs that lasted $500 \mathrm{~ms}$ was 191 considered as the maximal voluntary torque. During the first of the two MVCs, the LOA was 192 calculated as follows (Allen et al., 1995):

$$
\text { LOA }(\%)=[1-(\text { superimposed doublet/control doublet }) \times 100]
$$

194 The RMS of the soleus EMG activity ( $\mathrm{RMS}_{\mathrm{EMG}}$ ) was calculated during the maximal torque 195 plateau (as defined above) during each sequence of neuromuscular testing. The peak-to-peak 196 amplitudes of Msup, V-wave and Hsup were calculated. EMG $\mathrm{RMS}_{\text {, }}$ V-wave and Hsup were 197 normalized with respect to Msup to avoid any influence of peripheral changes on the central 198 parameters: $\mathrm{EMG}_{\mathrm{RMS}} /$ Msup, Hsup/Msup, V/Msup.

199 The area under the curve (AUC) of [HbO] and [HbR] was calculated and used as an index of 200 cortical activity (Shimodera et al., 2012). AUC of [HbO] and [HbR] from each channel over 
the M1, S1 and PFC areas was calculated and normalized over time from the beginning to the

202 end (labeled with the specific events set in the fNIRS software during recording) of the best of 203 the two MVCs performed during the neuromuscular tests. Data from the two channels over 204 M1 and from the two channels over S1 were then averaged, resulting respectively in an 205 overall response of the M1 and S1 areas.

\subsection{Statistical analysis}

All statistical analyses were performed using Statistica software (StatSoft, Inc., version 6.0, Tulsa, OK, USA). All variables recorded before, during and after the NMES protocol (or resting periods for pre-experiment check-up) were tested using a one-way repeated measure ANOVA (time effect): before NMES (pre), after 17 trains (post17), after 34 trains (post34), after 51 trains (post51), after 68 trains (post68) and after 85 trains (post85). The underlying assumptions of ANOVA were checked using Skewness-Kurtosis coefficients (normality of distribution) and the Mauchly test (sphericity of variance). When the ANOVA F ratio was significant $(p<0.05)$, means were compared by a LSD post-hoc test. Data are reported as 216 means and standard deviation (SD) unless specified.

\section{Results}

\subsection{Pre-experiment check-up}

221 The torque recorded during MVCs did not change significantly throughout the control 222 condition $(F=0.62, p=0.68)$. Likewise, there was no significant changes nor in LOA neither in evoked potential properties (all $p>0.15$ ). Furthermore, the cortical activity remained unchanged during the control session over the M1 and S1 areas (all $p>0.31$ ). 
3.2. Stimulation trains and maximal voluntary contraction torques

227 The torque evoked during the stimulation trains decreased during the NMES protocol $(F=16.2, p<0.001)$. The decrease was significant from post17 $(p<0.01)$ and remained significant until the end of the fatigue protocol ( -8.2 to $-14.8 \%$ between trains $15-17$ to trains 83-85; Fig. 3A).

231 The torque produced during MVCs decreased throughout the NMES protocol $(F=21.5$, $232 p<0.001)$. The decrease was significant from post17 $(p<0.001)$ and remained significant until 233 the end of the protocol (-10.8 to $-19.9 \%$ between post34 and post 85 ; Fig. 3B).

3.3. LOA, mechanical response and evoked potentials

236 Detailed results of the LOA, mechanical response and evoked potentials are given in Table 1. 237 A significant decrease in LOA $(F=9.06, p<0.001)$ was observed immediately after the first 17 238 NMES trains $(p<0.01)$. The Pt values decreased significantly $(F=16.28, p<0.001)$ from post34 239 ( $\mathrm{p}<0.001)$. Msup amplitude decreased significantly during the NMES protocol $(F=12.27$, $240 p<0.001)$ from post17 $(p<0.001)$. $\mathrm{EMG}_{\mathrm{RMS}} / \mathrm{Msup}$ declined significantly during the 241 experimental session $(F=3.4, p<0.01)$ from post34 $(\mathrm{p}<0.01)$. V/Msup exhibited a significant 242 decrease $(F=2.78, p<0.05)$ immediately at post17 $(\mathrm{p}<0.05)$. Hsup/Msup was not significantly modified $(F=1.135, p=0.35)$.

\subsection{Cortical activity}

246 A significant decrease in $[\mathrm{HbO}]$ and increase in $[\mathrm{HbR}]$ were found over the $\mathrm{M} 1$ area during 247 the experimental session $(\mathrm{F}=8.49, p<0.001$ and $\mathrm{F}=4.03, p<0.01$, respectively). These 248 significant modifications of $[\mathrm{HbO}]$ and $[\mathrm{HbR}]$ occurred at post17 $(p<0.001$ and $p<0.05)$ and 249 persisted until the end of the protocol (Fig. 4A). 
250 A comparable result ([HbO] decrease and $[\mathrm{HbR}]$ increase) was found over the $\mathrm{S} 1$ area $251(\mathrm{~F}=3.85, p<0.01$ and $\mathrm{F}=2.63, p<0.05$, respectively). The decrease in [HbO] was significant at 252 post $17(p<0.05)$, while $[\mathrm{HbR}]$ increased significantly from post34 $(p<0.01)$ (Fig. 4B).

253 Over the PFC area, [HbO] was $1.17 \pm 0.31,1.3 \pm 0.32,1.27 \pm 0.31,0.8 \pm 0.22,0.97 \pm 0.19$ and $2540.76 \pm 0.33 \mu \mathrm{M}$ at pre, post17, post 34 , post51, post68 and post85, respectively, and was not 255 significantly modified $(\mathrm{F}=1.43, p=0.24)$. [HbR] was $-0.13 \pm 0.13,-0.06 \pm 0.07,-0.04 \pm 0.8$, $2560.03 \pm 0.07,-0.16 \pm 0.12$ and $-0.07 \pm 0.14 \mu \mathrm{M}$ at pre, post17, post 34 , post 51 , post68 and post85, 257 respectively, and was not significantly modified throughout the experimental session $(\mathrm{F}=1.14$, $258 p=0.35)$.

260 Figure 5 represents raw data of LOA, Msup, V-wave, Hsup and fNIRS signal from one 261 individual participant at pre and post85.

\section{Discussion}

The present study aimed to identify the supra-spinal factors involved in neuromuscular fatigue 266 induced by low frequency stimulation of the plantar flexors. The results showed the development of neuromuscular fatigue, as evidenced by the significant MVC torque decrease, which was accompanied by changes occurring at the supra-spinal level. The major finding of

269 the study was an early and progressive decline in cortical activity over the M1 and S1 areas 270 during MVCs following NMES trains.

272 Since the pre-experiment check-up did not reveal any parameters modifications (maximal 273 voluntary torque, superimposed evoked potential and cortical activity), it can be assert that the 274 NMES protocol caused all the identifying results in the experiment. 
275 The neuromuscular fatigue induced by the NMES protocol was evidenced by the decrease in 276 maximal voluntary torque. This torque alteration may have originated at peripheral, spinal or 277 supra-spinal levels. Regarding the peripheral level, a decrease in Msup and peak twitch 278 amplitude was observed, consistent with neuromuscular excitability and muscle contractility 279 failure, respectively. These results support other studies investigating higher stimulation 280 frequencies $(75 \mathrm{~Hz})$ and/or other muscular groups (Boerio et al. 2005; Papaiordanidou et al. 281 2010a; Zory et al. 2005).

282 Confirming previous studies (Boerio et al., 2005, Papaiordanidou et al., 2010b), 283 neuromuscular fatigue could not be ascribed to spinal excitability alterations since the 284 Hsup/Msup ratio did not change. Conversely, $\mathrm{LOA}$ and $\mathrm{EMG}_{\mathrm{RMS}} / \mathrm{Msup}$ decreased throughout 285 the protocol. The decrease in these peripheral indices of the descending neural drive is 286 consistent with a drop in the central motor drive. In accordance with earlier conclusions on 287 the nature of the CNS alterations under NMES (Boerio et al., 2005, Papaiordanidou et al., 288 2010b), and because in the present study no modification in spinal excitability was found, the 289 decrease in the central motor drive most probably was derived from the supra-spinal level.

290 As in a previous study conducted at higher frequencies (Laurin et al., 2012), we observed a 291 V/Msup decrease, a change in a variable that likely reflects both spinal excitability (Crone \& 292 Nielsen, 1994) and descending neural drive (Aagaard et al., 2002). Because spinal excitability 293 was not modified, it is assumed that the reported V/Msup decline was instead caused by a 294 decrease in the descending neural drive upstream the spinal level. Thus, the V/Msup kinetic is 295 in line with the involvement of supra-spinal impairments in decreasing the central motor drive 296 under NMES.

297 Taken individually, electrophysiological parameters like V/Msup, LOA and $\mathrm{EMG}_{\mathrm{RMS}} / \mathrm{Msup}$ 298 cannot provide information on the supra-spinal mechanisms responsible for torque decline. 299 Furthermore, because they are recorded at the peripheral level, the decrease in central motor 
drive reflected by these markers, even when normalized over spinal and muscle properties, is

301 based only on an extrapolation. In the current study, we used a new complementary approach

302 to assess supra-spinal alterations by combining the results derived from classical

303 electrophysiological data with neuroimaging. The fNIRS data exhibited a decrease in [HbO]

304 and an increase in [HbR] over the M1 and S1 areas as neuromuscular fatigue developed, a

305 typical sign of decreased neural activity (Wenzel et al., 2000). These results, which

306 corroborate the electrophysiological data, are consistent with an immediate decrease in neural

307 activity from the first NMES sequence. Furthermore, taken together, they provide the first

308 evidence that a cortical motor output decline is implicated in the loss in voluntary force

309 production after NMES.

310 The PFC area did not exhibit any changes in [HbO] or [HbR] throughout the fatiguing

311 protocol. This area is notably involved in motivation (Kouneiher et al., 2009) and is

312 considered as the main facilitation structure of the brain (Tanaka \& Watanabe, 2012). Other

313 studies have reported a decrease in $[\mathrm{HbO}]$ within the PFC area during voluntary whole-body

314 exercise just before task failure, suggesting its possible involvement in decreasing neural

315 drive (Rupp \& Perrey, 2008, Shibuya et al., 2004). The same pattern could not be found with

316 electrically induced fatigue, thus the decrease in cortical motor output cannot be ascribed to

317 alterations in motivation or facilitation processes from the PFC area during this specific task.

318 One of the objectives of the study was to better understand the supra-spinal mechanisms

319 responsible for voluntary muscle torque decline after NMES. Our initial hypothesis was that

320 an increase in inhibitory input from the S1 to the M1 area would explain the decrease in

321 cortical motor output. Because the activation of inhibitory interneurons increases local energy

322 consumption (Jueptner \& Weiller, 1995), areas involved in the onset of motor output

323 inhibition should appear overactivated. In the current study, both the M1 and S1 areas were

324 deactivated, so it can be assumed that the onset of the inhibition process took place upstream 
of these two primary cortical areas. It is thus possible to suggest the involvement of deeper structures. The thalamus, the insular and the cingulate cortex should be considered, as their implication in the inhibition of central motor drive has been clearly suggested during volitional fatigue (Tanaka et al., 2013, Hilty et al., 2011a, Jouanin et al., 2009).

The decrease in cortical activation over the M1 and S1 areas was evident from the very first

330 NMES sequence. Previous studies dealing with voluntary fatigue contractions reported a 331 different activation pattern, showing an initial increase in cortical activity followed by a final 332 decrease in cortical activity near the exhaustion threshold (Liu et al., 2002, Shibuya \& Tachi, 333 2006, Shibuya \& Kuboyama, 2010). The early decrease in cortical activity after NMES is in 334 accordance with the characteristics of electrically induced contractions as opposed to 335 voluntary contractions. Here, we suggest that the higher metabolic stress induced by NMES 336 (Jubeau et al., 2012, Theurel et al., 2007) led to an early elevated firing rate of the group III and IV afferents, which in turn caused the recruitment of inhibitory interneurons within the 338 brain to decrease the central motor drive.

340 Methodological consideration:

341 We did not assess the gastrocnemius EMG activity. At $110^{\circ}$-knee joint angle, the contribution 342 of the gastrocnemius muscles to plantar flexor torque should be between $30 \%$ and $40 \%$ 343 (Cresswell et al., 1995; Fukunaga et al., 1992). After the electrically-induced fatigue, we 344 observed a decline in the plantar flexor torque without the possibility to identify the 345 contribution of each muscle in the declining torque. Although we could only report a decline 346 of the soleus $\mathrm{EMG}_{\mathrm{RMS}}$ activity, a similar decline in the gastrocnemius musles is strongly expected (Papaiordanidou et al. 2010b).

348 The superimposed evoked potentials during MVCs were elicited on the tibial nerve using 349 stimulation intensities determined at rest. By doing so, activity-dependent changes in motor 
axonal excitability during MVCs may have led to unsuitable stimulation intensities. To

351 overcome this issue, the maximal intensity to elicit Msup was increased by $20 \%$. Indeed, it 352 has been shown that the plateau of the maximal superimposed M-wave during MVC is 353 obtained by stimulation of at least $120 \%$ of the maximal M-wave determined at rest (Racinais 354 et al., 2013). Conversely, we did not increase the submaximal intensity to elicit superimposed 355 H-waves during MVCs. By using intensity determined at rest, the superimposed H-waves 356 obtained in the study were presumably not maximal but were rather on the ascending part of 357 the recruitment curve (Racinais et al., 2013). Yet, it has been shown that the reliability of the 358 superimposed H-reflex is higher when assessed in the ascending part of the recruitment curve 359 (Grospretre \& Martin, 2012).

360 LOA and superimposed evoked potentials are subjects to variability and were recorded only 361 once during each neuromuscular tests session. However, no changes were found in the control condition for any recorded parameter, supporting that NMES rather than variability was likely responsible for LOA and superimposed evoked potentials changes.

\section{Conclusion}

366 In conclusion, the present study provides evidence that a cortical motor output decrease is 367 involved in the loss of force after NMES at low frequencies. The decrease in central motor 368 drive was demonstrated from the very first NMES sequence by the consistency between the 369 kinetics of the electrophysiological and the fNIRS-measured hemodynamics data over the M1 370 area. The fact that the S1 activity also decreased after NMES indicates that S1 is not involved 371 in the inhibition of the cortical motor output during this specific task. 
374 The authors would like to thank Prof. Stephane PERREY for the use of NIRS equipment

375 funded by a grant in aid from the Languedoc-Roussillon Region council (AVENIR).

376

377 Conflict of interest

378 No conflict of interest, financial or otherwise, is declared by the authors. 


\section{REFERENCES}

Aagaard, P., Simonsen, E. B., Andersen, J. L., Magnusson, P. \& Dyhre-Poulsen, P. 2002. Neural adaptation to resistance training: changes in evoked V-wave and H-reflex responses. J Appl Physiol (1985), 92, 2309-18.

Allen, G. M., Gandevia, S. C. \& McKenzie, D. K. 1995. Reliability of measurements of muscle strength and voluntary activation using twitch interpolation. Muscle Nerve, 18, 593-600.

Amann, M., Proctor, L. T., Sebranek, J. J., Pegelow, D. F. \& Dempsey, J. A. 2009. Opioidmediated muscle afferents inhibit central motor drive and limit peripheral muscle fatigue development in humans. $J$ Physiol, 587, 271-83.

Boerio, D., Jubeau, M., Zory, R. \& Maffiuletti, N. A. 2005. Central and peripheral fatigue after electrostimulation-induced resistance exercise. Med Sci Sports Exerc, 37, 973-8.

Chen, R., Corwell, B. \& Hallett, M. 1999. Modulation of motor cortex excitability by median nerve and digit stimulation. Exp Brain Res, 129, 77-86.

Colier, W. N., Quaresima, V., Oeseburg, B. \& Ferrari, M. 1999. Human motor-cortex oxygenation changes induced by cyclic coupled movements of hand and foot. Exp Brain Res, 129, 457-61.

Cresswell, A. G., Loscher, W. N. \& Thorstensson, A. 1995. Influence of gastrocnemius muscle length on triceps surae torque development and electromyographic activity in man. Exp Brain Res, 105, 283-90.

Crone, C. \& Nielsen, J. 1994. Central control of disynaptic reciprocal inhibition in humans. Acta Physiol Scand, 152, 351-63.

Delpy, D. T., Cope, M., van der Zee, P., Arridge, S., Wray, S. \& Wyatt, J. 1988. Estimation of optical pathlength through tissue from direct time of flight measurement. Phys Med Biol, 33, 1433-42.

Derosiere, G., Alexandre, F., Bourdillon, N., Mandrick, K., Ward, T. E. \& Perrey, S. 2014. Similar scaling of contralateral and ipsilateral cortical responses during graded unimanual force generation. Neuroimage, 85 Pt 1, 471-7.

Doucet, B. M., Lam, A. \& Griffin, L. 2012. Neuromuscular electrical stimulation for skeletal muscle function. Yale J Biol Med, 85, 201-15.

Downey, R. J., Bellman, M., Sharma, N., Wang, Q., Gregory, C. M. \& Dixon, W. E. 2011. A novel modulation strategy to increase stimulation duration in neuromuscular electrical stimulation. Muscle Nerve, 44, 382-7.

Duncan, A., Meek, J. H., Clemence, M., Elwell, C. E., Fallon, P., Tyszczuk, L., Cope, M. \& Delpy, D. T. 1996. Measurement of cranial optical path length as a function of age using phase resolved near infrared spectroscopy. Pediatr Res, 39, 889-94.

Forss, N., Hari, R., Salmelin, R., Ahonen, A., Hamalainen, M., Kajola, M., Knuutila, J. \& Simola, J. 1994. Activation of the human posterior parietal cortex by median nerve stimulation. Exp Brain Res, 99, 309-15.

Fox, P. T., Raichle, M. E., Mintun, M. A. \& Dence, C. 1988. Nonoxidative glucose consumption during focal physiologic neural activity. Science, 241, 462-4.

Fuglevand, A. J. \& Keen, D. A. 2003. Re-evaluation of muscle wisdom in the human adductor pollicis using physiological rates of stimulation. $J$ Physiol, 549, 865-75.

Fukunaga, T., Roy, R. R., Shellock, F. G., Hodgson, J. A., Day, M. K., Lee, P. L., Kwong-Fu, H. \& Edgerton, V. R. 1992. Physiological cross-sectional area of human leg muscles based on magnetic resonance imaging. J Orthop Res, 10, 928-34.

Garland, S. J. \& Gossen, E. R. 2002. The muscular wisdom hypothesis in human muscle fatigue. Exerc Sport Sci Rev, 30, 45-9. 
Gorgey, A. S., Black, C. D., Elder, C. P. \& Dudley, G. A. 2009. Effects of electrical stimulation parameters on fatigue in skeletal muscle. J Orthop Sports Phys Ther, 39, 684-92.

Grospretre, S. \& Martin, A. 2012. H reflex and spinal excitability: methodological considerations. J Neurophysiol, 107, 1649-54.

Hilty, L., Jancke, L., Luechinger, R., Boutellier, U. \& Lutz, K. 2011a. Limitation of physical performance in a muscle fatiguing handgrip exercise is mediated by thalamo-insular activity. Hum Brain Mapp, 32, 2151-60.

Hilty, L., Lutz, K., Maurer, K., Rodenkirch, T., Spengler, C. M., Boutellier, U., Jancke, L. \& Amann, M. 2011b. Spinal opioid receptor-sensitive muscle afferents contribute to the fatigue-induced increase in intracortical inhibition in healthy humans. Exp Physiol, 96, 505-17.

Hlushchuk, Y. \& Hari, R. 2006. Transient suppression of ipsilateral primary somatosensory cortex during tactile finger stimulation. JNeurosci, 26, 5819-24.

Hoffman, M., Schrader, J., Applegate, T. \& Koceja, D. 1998. Unilateral postural control of the functionally dominant and nondominant extremities of healthy subjects. $J$ Athl Train, 33, 319-22.

Hultman, E., Sjoholm, H., Jaderholm-Ek, I. \& Krynicki, J. 1983. Evaluation of methods for electrical stimulation of human skeletal muscle in situ. Pflugers Arch, 398, 139-41.

Huppert, T. J., Diamond, S. G., Franceschini, M. A. \& Boas, D. A. 2009. HomER: a review of time-series analysis methods for near-infrared spectroscopy of the brain. Appl Opt, 48, D280-98.

Jouanin, J. C., Peres, M., Ducorps, A. \& Renault, B. 2009. A dynamic network involving M1S1, SII-insular, medial insular, and cingulate cortices controls muscular activity during an isometric contraction reaction time task. Hum Brain Mapp, 30, 675-88.

Jubeau, M., Muthalib, M., Millet, G. Y., Maffiuletti, N. A. \& Nosaka, K. 2012. Comparison in muscle damage between maximal voluntary and electrically evoked isometric contractions of the elbow flexors. Eur J Appl Physiol, 112, 429-38.

Jueptner, M. \& Weiller, C. 1995. Review: does measurement of regional cerebral blood flow reflect synaptic activity? Implications for PET and fMRI. Neuroimage, 2, 148-56.

Keenan, K. G., Farina, D., Maluf, K. S., Merletti, R. \& Enoka, R. M. 2005. Influence of amplitude cancellation on the simulated surface electromyogram. J Appl Physiol (1985), 98, 120-31.

Kooistra, R. D., de Ruiter, C. J. \& de Haan, A. 2007. Conventionally assessed voluntary activation does not represent relative voluntary torque production. Eur J Appl Physiol, 100, 309-20.

Kouneiher, F., Charron, S. \& Koechlin, E. 2009. Motivation and cognitive control in the human prefrontal cortex. Nat Neurosci, 12, 939-45.

Laurin, J., Dousset, E., Carrivale, R., Grelot, L. \& Decherchi, P. 2012. Recovery pattern of motor reflex after a single bout of neuromuscular electrical stimulation session. Scand J Med Sci Sports, 22, 534-44.

Liu, J. Z., Dai, T. H., Sahgal, V., Brown, R. W. \& Yue, G. H. 2002. Nonlinear cortical modulation of muscle fatigue: a functional MRI study. Brain Res, 957, 320-9.

Maffiuletti, N. A. 2010. Physiological and methodological considerations for the use of neuromuscular electrical stimulation. Eur J Appl Physiol, 110, 223-34.

Papaiordanidou, M., Guiraud, D. \& Varray, A. 2010a. Does central fatigue exist under lowfrequency stimulation of a low fatigue-resistant muscle? Eur J Appl Physiol, 110, 81523.

Papaiordanidou, M., Guiraud, D. \& Varray, A. 2010b. Kinetics of neuromuscular changes during low-frequency electrical stimulation. Muscle Nerve, 41, 54-62. 
Racinais, S., Maffiuletti, N. A. \& Girard, O. 2013. M-wave, H- and V-reflex recruitment curves during maximal voluntary contraction. J Clin Neurophysiol, 30, 415-21.

Rupp, T. \& Perrey, S. 2008. Prefrontal cortex oxygenation and neuromuscular responses to exhaustive exercise. Eur J Appl Physiol, 102, 153-63.

Shibuya, K. \& Kuboyama, N. 2010. Decreased activation in the primary motor cortex area during middle-intensity hand grip exercise to exhaustion in athlete and nonathlete participants. Percept Mot Skills, 111, 19-30.

Shibuya, K. \& Tachi, M. 2006. Oxygenation in the motor cortex during exhaustive pinching exercise. Respir Physiol Neurobiol, 153, 261-6.

Shibuya, K., Tanaka, J., Kuboyama, N., Murai, S. \& Ogaki, T. 2004. Cerebral cortex activity during supramaximal exhaustive exercise. J Sports Med Phys Fitness, 44, 215-9.

Shimodera, S., Imai, Y., Kamimura, N., Morokuma, I., Fujita, H., Inoue, S. \& Furukawa, T. A. 2012. Mapping hypofrontality during letter fluency task in schizophrenia: a multichannel near-infrared spectroscopy study. Schizophr Res, 136, 63-9.

Sutherland, M. T. 2006. The hand and the ipsilateral primary somatosensory cortex. $J$ Neurosci, 26, 8217-8.

Tanaka, M., Ishii, A. \& Watanabe, Y. 2013. Neural correlates of central inhibition during physical fatigue. PLoS One, 8, e70949.

Tanaka, M. \& Watanabe, Y. 2012. Supraspinal regulation of physical fatigue. Neurosci Biobehav Rev, 36, 727-34.

Theurel, J., Lepers, R., Pardon, L. \& Maffiuletti, N. A. 2007. Differences in cardiorespiratory and neuromuscular responses between voluntary and stimulated contractions of the quadriceps femoris muscle. Respir Physiol Neurobiol, 157, 341-7.

Tokimura, H., Di Lazzaro, V., Tokimura, Y., Oliviero, A., Profice, P., Insola, A., Mazzone, P., Tonali, P. \& Rothwell, J. C. 2000. Short latency inhibition of human hand motor cortex by somatosensory input from the hand. J Physiol, 523 Pt 2, 503-13.

Wenzel, R., Wobst, P., Heekeren, H. H., Kwong, K. K., Brandt, S. A., Kohl, M., Obrig, H., Dirnagl, U. \& Villringer, A. 2000. Saccadic suppression induces focal hypooxygenation in the occipital cortex. Journal of cerebral blood flow and metabolism : official journal of the International Society of Cerebral Blood Flow and Metabolism, 20, 1103-10. 
Tables:

Table 1. EMG activity, voluntary activation level, superimposed evoked potentials and mechanical responses.

\begin{tabular}{lllllll}
\hline & Pre & Post 17 & Post 34 & Post 51 & Post 68 & Post 85 \\
\hline LOA (\%) & $93.33(1.31)$ & $87.96(1.82)^{* *}$ & $87.69(1.97)^{* * *}$ & $86.27(2.03)^{* * *}$ & $84.3(2.81)^{* * *}$ & $83.5(2.59)^{* * *}$ \\
Pt (Nm) & $21.53(1.1)$ & $21.38(1.08)$ & $20.23(0.96)^{* * *}$ & $19.92(0.95)^{* * *}$ & $19.44(0.88)^{* * *}$ & $19.28(0.81)^{* * *}$ \\
Msup (mV) & $10.9(1)$ & $9.13(0.91)^{* * *}$ & $9.13(0.91)^{* * *}$ & $8.63(0.88)^{* * *}$ & $8.82(0.9)^{* * *}$ & $8.27(0.73)^{* * *}$ \\
EMG $_{\mathrm{RMS}}$ Msup & $0.0224(0.002)$ & $0.0223(0.002)$ & $0.0198(0.001)^{*}$ & $0.0204(0.001)^{* *}$ & $0.0198(0.001)^{*}$ & $0.0203(0.001)^{*}$ \\
Hsup/Msup & $0.398(0.03)$ & $0.455(0.046)$ & $0.414(0.036)$ & $0.396(0.034)$ & $0.355(0.036)$ & $0.413(0.038)$ \\
V/Msup & $0.321(0.025)$ & $0.237(0.031)^{*}$ & $0.252(0.027)^{*}$ & $0.218(0.038)^{* *}$ & $0.227(0.031)^{* *}$ & $0.220(0.035)^{* *}$
\end{tabular}

Values are mean $(\mathrm{SE}){ }^{*} \mathrm{p}<0.05, * * \mathrm{p}<0.01$ and $* * * \mathrm{p}<0.001$ significantly different from pre values. 
Figure:

Fig. 1. Schematic representation of the experimental session. (A) Experimental design. (B) Decomposition of a sequence of neuromuscular tests performed after each sequence of trains. N.T: Neuromuscular Tests. Details on resting evoked potentials are given in electrically evoked potentials section.

A
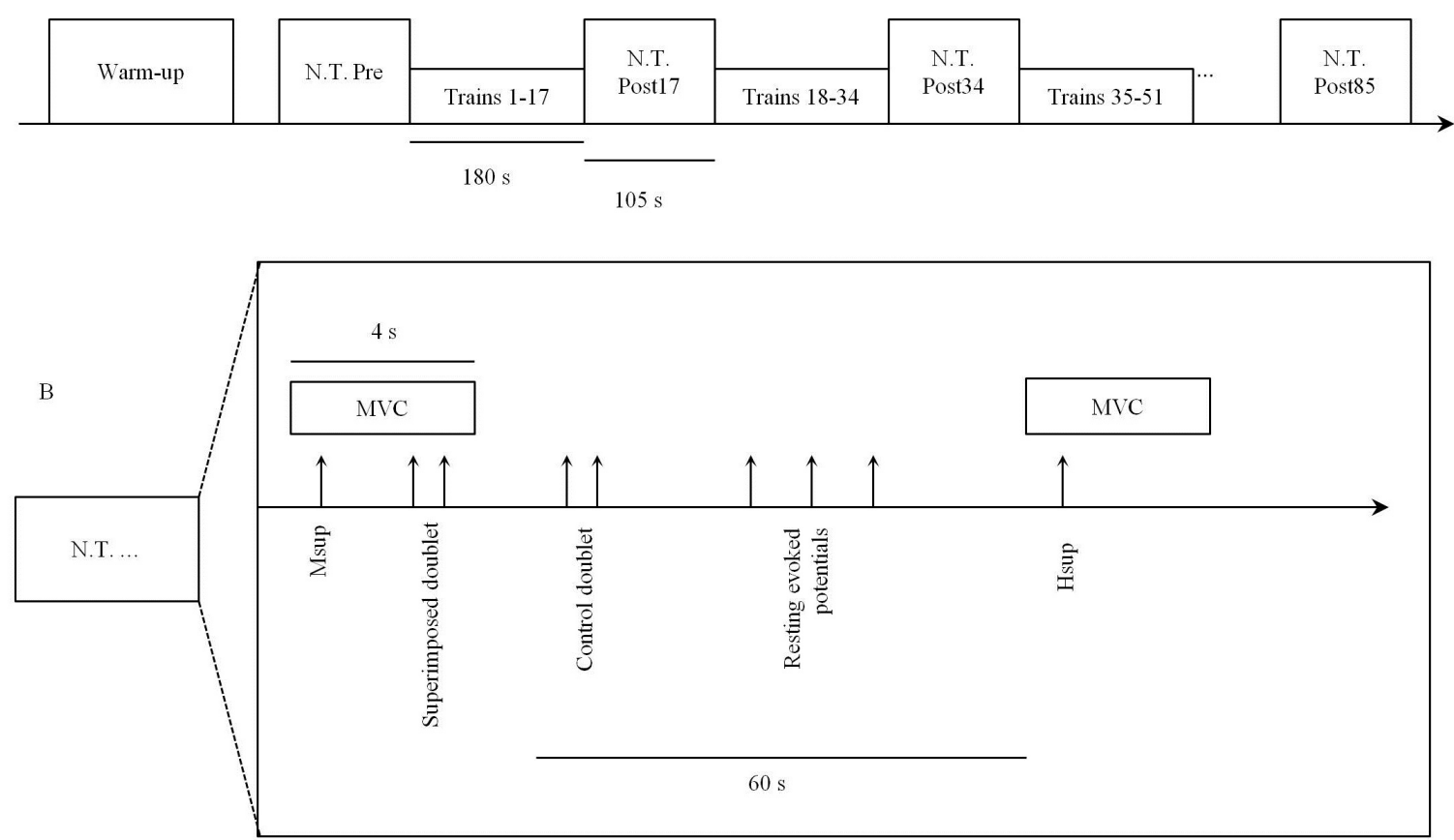
Fig. 2. Schematic representations of the placement of the fNIRS optodes. The emitors (E) were placed at $3.5 \mathrm{~cm}$ from the receptor $(\mathrm{R})$. The optodes were positioned according to the extended EEG 10-20 system: around $\mathrm{C}_{3}$ and $\mathrm{CP}_{3}$ (or $\mathrm{C}_{4}$ and $\mathrm{CP}_{4}$ ) to target the primary motor and somatosenry cortex, around $\mathrm{CP}_{1}$ (or $\mathrm{CP}_{2}$ ) to target the prefrontal cortex. $\mathrm{C}_{\mathrm{z}}$ is situated at the middle of the distance between the nasion and the inion. $\mathrm{CP}_{\mathrm{z}}$ is situated at $10 \%$ of the distance between the nasion and the inion. The optodes were placed on the contralateral side.

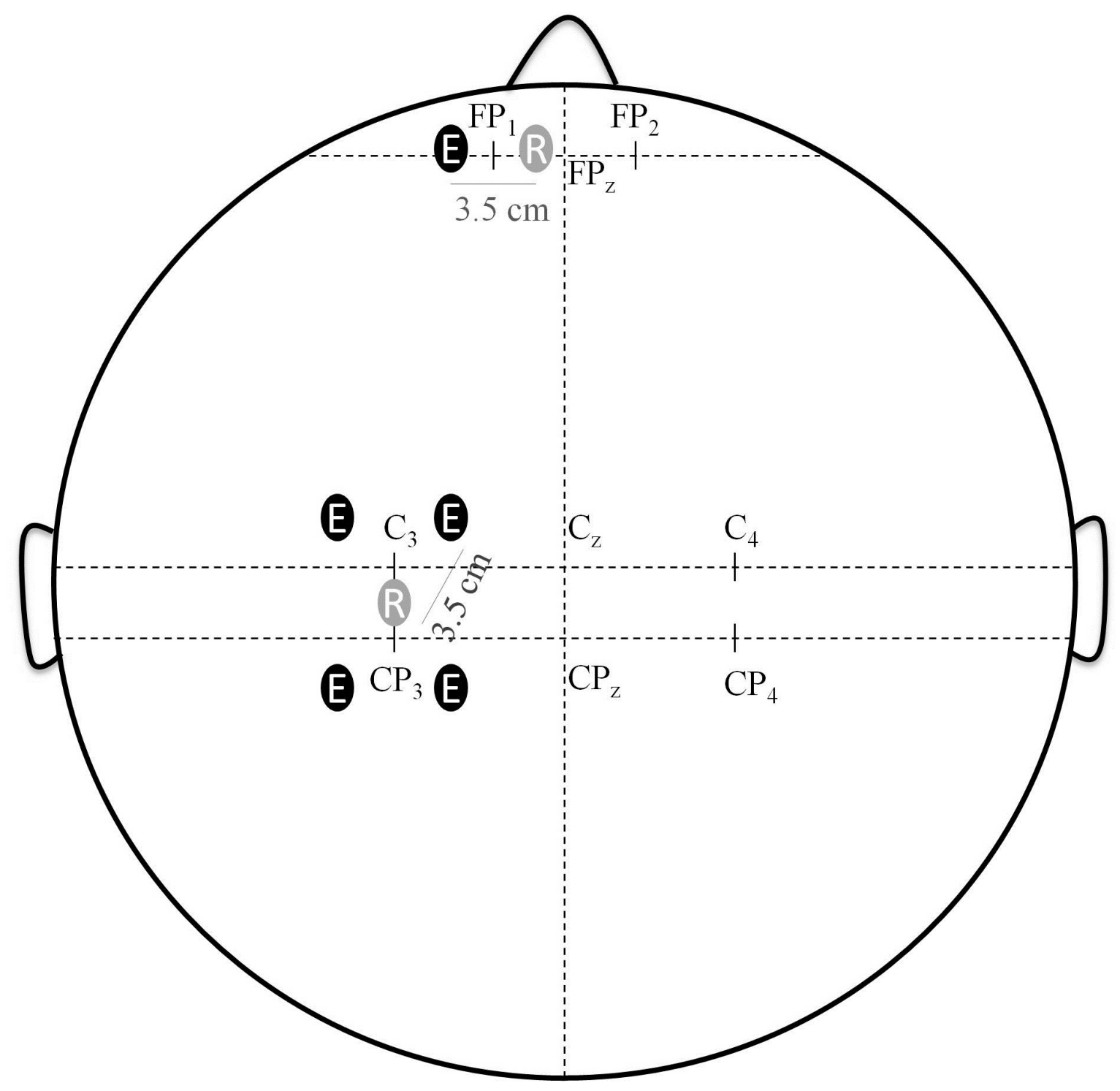


Fig. 3. (A) Torque evoked by the NMES trains. (B) Torque produced during maximal voluntary contractions. ${ }^{* *} p<0.01$ and ${ }^{* * *} p<0.001$ significantly different from pre values.

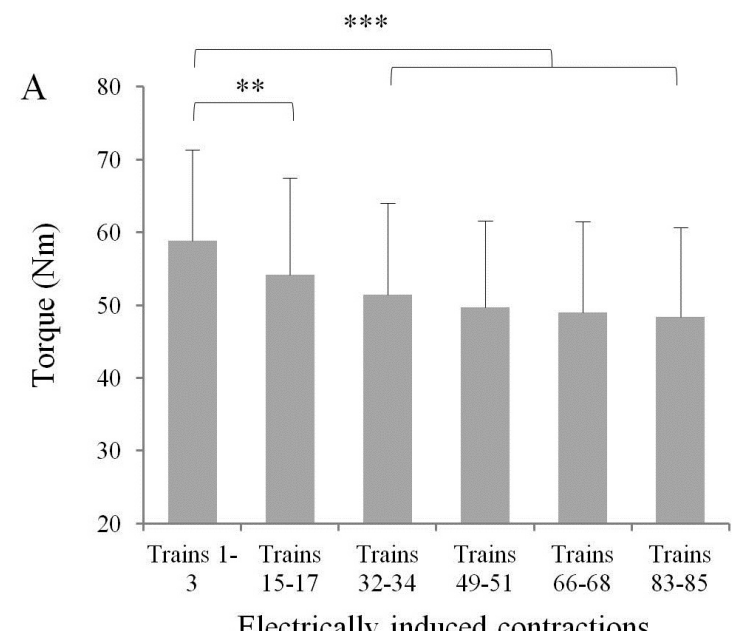

Electrically induced contractions

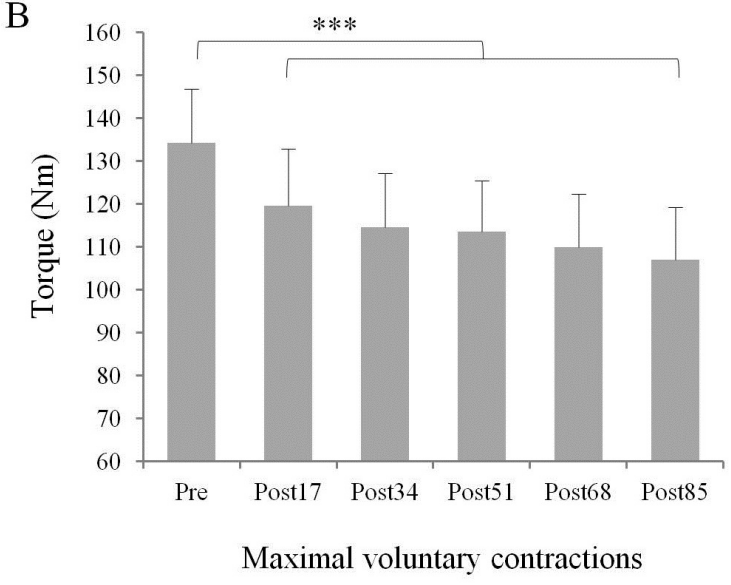


Fig. 4. (A) Primary motor cortex oxygenation. (B) Primary somatosensory cortex oxygenation. ${ }^{*} p<0.05, * * p<0.01$ and ${ }^{* * *} p<0.001$ significantly different from pre values. Vertical bars represent SE.

แ $[\mathrm{HbO}] \quad[\mathrm{HbR}]$
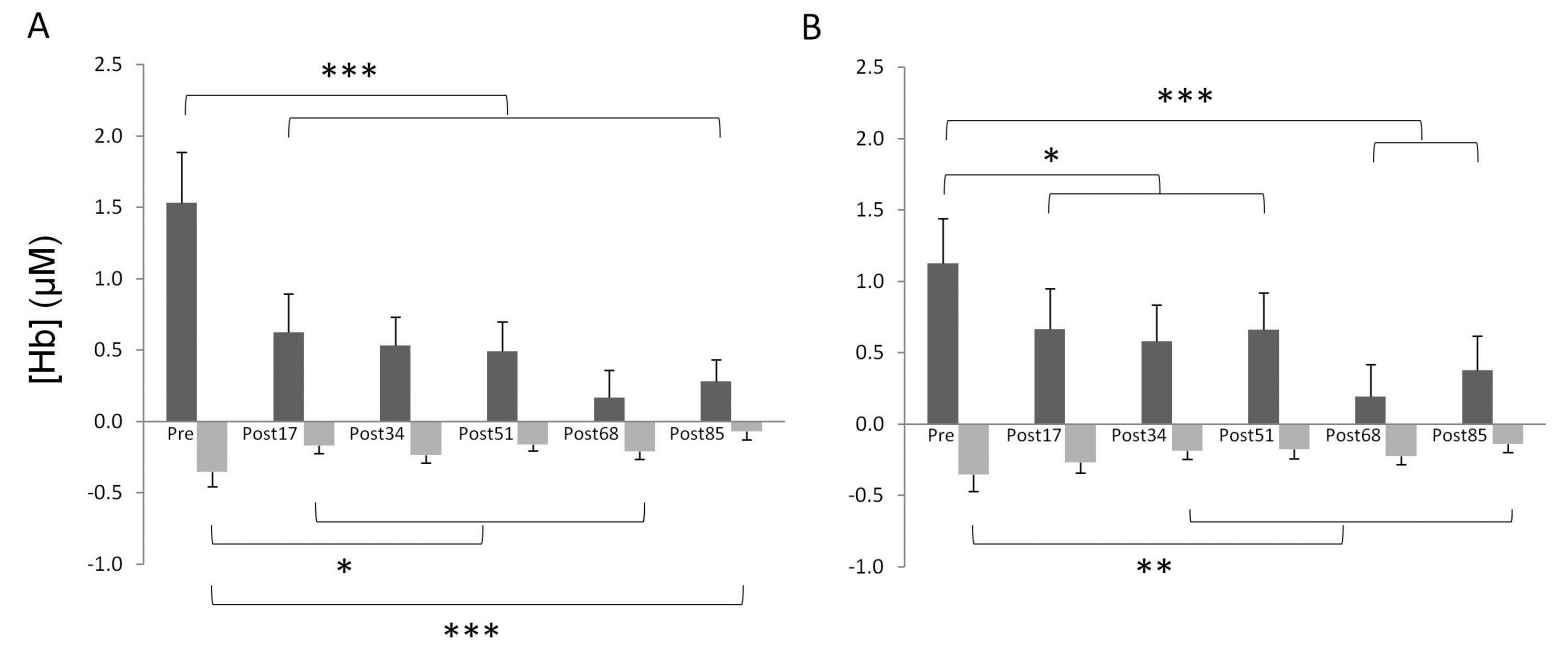
Fig. 5. Typical response of one subject for superimposed evoked potentials during maximal voluntary contractions (Msup, V-wave and Hsup), level of voluntary activation (twitch-like increment in torque) and cortical activity changes over the $\mathrm{M} 1$ area ( $\mathrm{HbO}$ and $\mathrm{HbR}$ ), before (pre) and after NMES (post85). Arrows indicate tibial nerve stimulation.
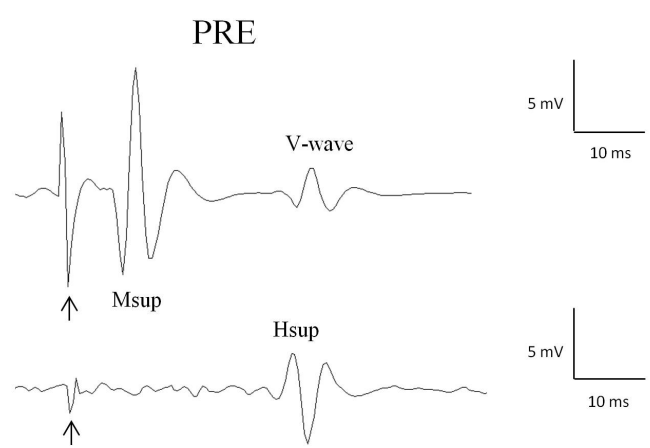

POST85
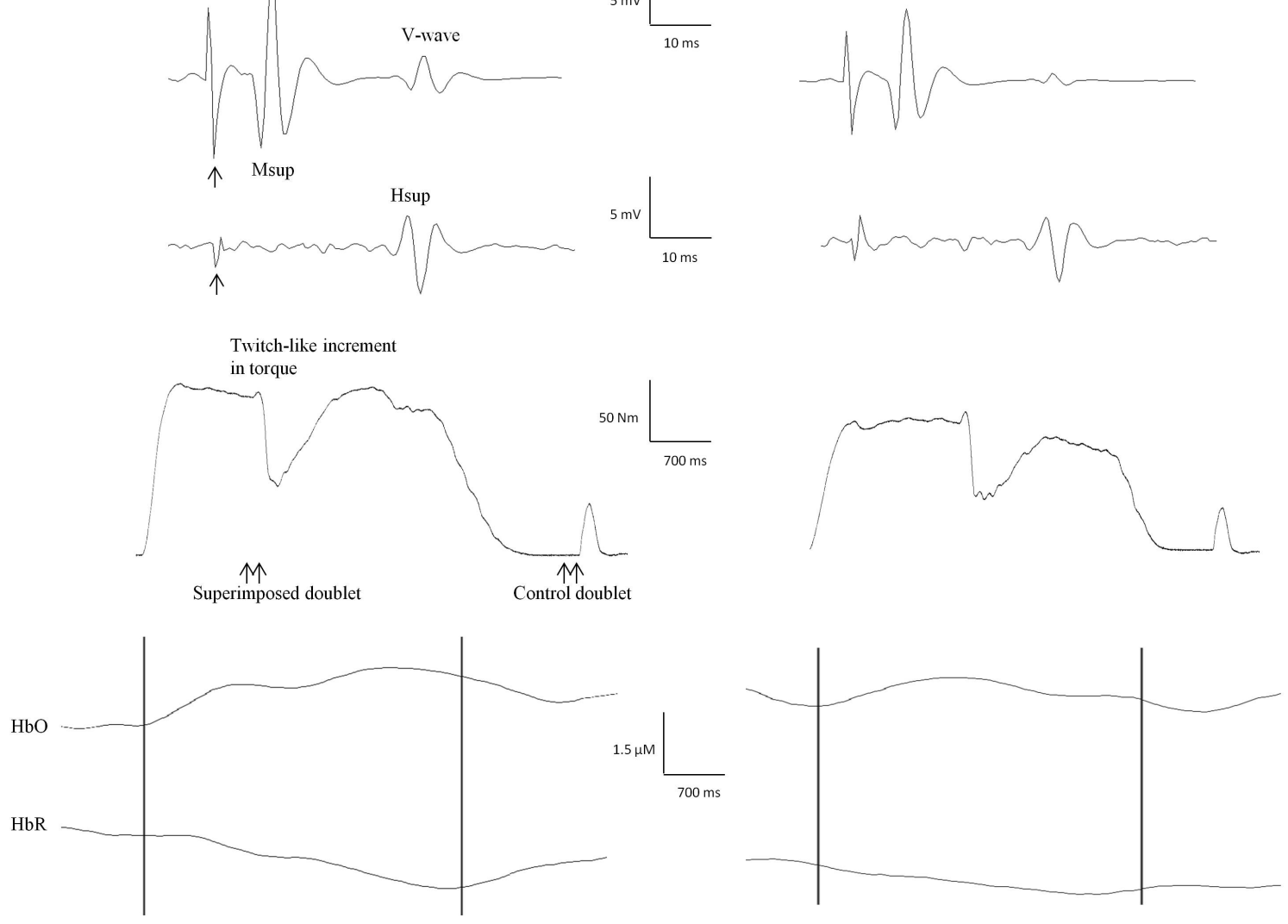